\title{
Fragilidade osmótica eritrocitária em gatos acometidos por hepatopatias e gatos com insuficiência renal
}

\author{
Erythrocyte osmotic fragility in cats with hepatic disease and cats with renal failure
}

\author{
Fernanda Elias $^{1}$ Sílvia Regina Ricci Lucas ${ }^{2}$ Mitika Kuribayashi Hagiwara ${ }^{3}$ \\ Márcia Mery Kogika² Regina Mieko Sakata Mirandola ${ }^{4}$
}

\section{RESUMO}

A fragilidade osmótica eritrocitária (FOE) avalia a resistência osmótica das células vermelhas em concentrações decrescentes de solução salina. A resistência depende da forma, volume, tamanho, conteúdo de hemoglobina e meia vida dos eritrócitos, e pode ser alterada por vários fatores fisiológicos ou patológicos. Existem poucos relatos sobre a FOE em gatos. O objetivo deste estudo foi avaliar a FOE em gatos hígidos, com enfermidades hepáticas e naqueles com insuficiência renal. Foram estudados 27 animais divididos em três grupos. A hemólise $50 \%$ foi notada de forma similar em todos os grupos. Em 100\% de hemólise, os eritrócitos de gatos com insuficiência renal e doença hepática mostraram maior fragilidade osmótica, embora esses animais apresentassem maiores níveis de colesterol sérico quando comparados aos gatos sadios do grupo controle. $O$ achado de maior FOE mesmo com níveis elevados de colesterol pode estar associado a características metabólicas da espécie.

Palavras-chave: gatos, colesterol, enfermidade hepática, insuficiência renal, eritrócitos.

\section{ABSTRACT}

Erythrocyte osmotic fragility (EOF) evaluates the resistance of red blood cells to saline solution of decreasing concentrations. Cells resistance is related to their shape, volume and size, as well as to the hemoglobin content and half life and can be altered by physiologic or pathologic mecanisms. There are few descriptions and litlle is known about EOF in cats. To clear up this point, EOF of healthy cats, and those with liver disease and cats with renal failure were studied. Twenty-seven adult cats were divided in three groups. Hemolysis 50\% occurred similarly in all groups, although relevant differences in relation to $100 \%$ hemolysis were found. Erythrocyte from cats with renal failure and from icteric animals with hepatic disease showed higher osmotic fragility and elevated cholesterol serum levels when compared to those belonging to the control group. These findings may be associated to the metabolic characteristics of this specie.

Key words: cats, cholesterol, liver disease, renal failure, erythrocyte.

\section{INTRODUÇÃO}

A fragilidade osmótica eritrocitária (FOE) pode ser definida como a resistência dos eritrócitos à hemólise, avaliada pelo uso de soluções tamponadas de $\mathrm{NaCl}$ em água destilada em concentrações decrescentes de $0,85 \%$ a $0 \%$ (JAIN, 1986). O controle do volume celular através da eliminação ativa de solutos é um dos mecanismos pelo qual a lise da membrana eritrocitária é evitada in vivo (MAKINDE \& BOBADE, 1994). As células, quando suspensas em meio hipotônico, aumentam até atingir um volume crítico de hemólise antes de serem lisadas (Ponder apud JAIN, 1973). Assim sendo, a fragilidade celular varia conforme a concentração de sal e segue uma distribuição normal em animais sadios (JAIN, 1973), havendo, entretanto, diferenças entre as espécies (JAIN, 1986).

Vários fatores intrínsecos e extrínsecos influenciam a fragilidade osmótica dos eritrócitos, ocorrendo diminuição ou aumento desta em condições

\footnotetext{
${ }^{1}$ Médico Veterinário, Ex-Bolsista de Iniciação Científica do CNPq.

${ }^{2}$ Médico Veterinário, Professora Dra., Departamento de Clínica Médica, Faculdade de Medicina Veterinária e Zootecnia (FMVZ),

Universidade de São Paulo (USP). E-mail: srrlucas@usp.br Autor para correspondência

${ }^{3}$ Médico Veterinário, Professora Titular, Departamento de Clínica Médica, FMVZ, USP.

${ }^{4}$ Farmacêutico/Bioquímico, Departamento de Clínica Médica, FMVZ, USP.
} 
diversas (JAIN, 1986). Segundo PERK et al. (1964), a fragilidade osmótica é influenciada por fatores como a forma, o volume e o tamanho do eritrócito, o tipo e a quantidade de hemoglobina, as diferenças na viscoelasticidade das membranas e na composição química e estrutural das mesmas. Aparentemente, células menores apresentam capacidade limitada de expansão e atingem o volume hemolítico crítico mais precocemente. O tempo de vida da célula também é muito importante já que os eritrócitos senescentes são mais frágeis que os jovens e correspondem a aproximadamente $30 \%$ da população eritrocitária. Além disso, segundo JAIN (1973) e MAKINDE \& BOBADE (1994), podem ocorrer variações fisiológicas (por exemplo, variações pós-prandiais) ou patológicas (presença de hematozoários, uremia, cirrose, processos auto-imunes, etc.).

Em pacientes humanos, o formato da célula é afetado por alterações na composição da membrana celular(WEED \& REED, 1966; TELEN \& KAUFMAN, 1999). Doenças associadas a alterações metabólicas como a insuficiência renal e a doença hepática, podem modificar a proporção de fosfolípides e de colesterol na membrana eritrocitária e conseqüentemente afetar a FOE. Os eritrócitos maduros não são capazes de sintetizar lipídios pela ausência da enzima acetil CoA carboxilase (PITTMAN \& MARTIN, 1966; TELEN \& KAUFMAN, 1999), com isso, a membrana celular sofre alterações em sua composição lipídica de acordo com as alterações dos lipídios circulantes (DIMOPOULLOS \& BEDELL, 1962; TELEN \& KAUFMAN, 1999).

Em felinos, a FOE é uma técnica pouco empregada, com poucas referências quanto aos seus valores em condições normais e nas diferentes afecções. Nessa espécie, a FOE parece não estar relacionada com o volume globular, nem com a percentagem de eritrócitos com corpúsculos de Heinz, mas sabe-se que ela está aumentada nas anemias hemolíticas auto-imunes e na hemobartonelose (CHRISTOPHER, 1989; JAIN, 1986). MAEDE (1980) observou um aumento na fragilidade osmótica eritrocitária de gatos com hemobartonelose. KOHN et al. (2000) observaram aumento da fragilidade osmótica em gatos apresentando anemias imunomediadas e acreditam que a possibilidade de uma predisposição genética à destruição dos eritrócitos não pode ser excluída. As mudanças na concentração de colesterol e fosfolípides plasmáticos não foram correlacionados à FOE durante o período experimental, porém, durante a fase anêmica da doença, quando os eritrócitos mostraram-se mais frágeis, a concentração de lipídios na membrana celular caiu rapidamente (KOHN et al., 2000).
A observação de alterações presentes em determinadas afecções em pacientes humanos e cães motivou a realização deste estudo com o objetivo de comparar a fragilidade osmótica eritrocitária entre os felinos hígidos e aqueles acometidos por insuficiência renal ou por doença hepática.

\section{MATERIALEMÉTODOS}

Foram utilizadas amostras de sangue de 27 gatos, sem evidências de anemia infecciosa felina ou anemia imunomediada, distribuídos em três grupos:

Grupo I - Constituído por 10 felinos adultos, machos e fêmeas, clinicamente hígidos, mantidos no Centro de Estudos de Doenças de Cães e Gatos do Departamento de Clínica Médica da Faculdade de Medicina Veterinária e Zootecnia da Universidade de São Paulo.

Grupo II - Constituído por oito gatos, machos e fêmeas, com insuficiência renal crônica, provenientes do atendimento do Hospital Veterinário da FMVZ - USP.

Grupo III - Constituído por nove gatos, machos e fêmeas, ictéricos, sem doença hemolítica, acometidos por afecções hepáticas, provenientes do atendimento do Hospital Veterinário da FMVZ - USP.

De cada animal foram colhidos $5,0 \mathrm{ml}$ de sangue através de punção da veia jugular, sendo $2,0 \mathrm{ml}$ com EDTA sódico a $10 \%$ para realização do hemograma e avaliação da fragilidade osmótica eritrocitária e 3,0ml sem anticoagulante para obtenção de soro que foi utilizado nas provas bioquímicas.

\section{Exames laboratoriais}

As amostras de sangue e soro sangüíneo obtidas dos animais dos três grupos, foram submetidas aos seguintes exames:

Hemograma: contagem de células sangüíneas em contador automático Serono Baker 9010.

Análises bioquímicas: as determinações de uréia, creatinina, proteínas séricas totais, albumina, triglicérides, colesterol total, HDL-colesterol, alanina aminotransferase (ALT), aspartato aminotransferase (AST), fosfatase alcalina (FA) e gama-glutamil transferase (GGT) foram realizadas em analisador automático RA-100 - Bayer.

Fragilidade Osmótica Eritrocitária: a análise da fragilidade osmótica eritrocitária foi realizada no período máximo de 24 horas após a colheita do sangue, de acordo com o método de Parpart apud JAIN (1986), que mensura a estabilidade dos eritrócitos em solução de cloreto de sódio em concentrações variando de 0,85 a $0 \%$ e que permite definir três parâmetros: 
a) a resistência eritrocitária mínima, que representa a concentração em que se inicia a hemólise; b) a resistência máxima, ou seja, a concentração de $\mathrm{NaCl}$ correspondente à hemólise total (hemólise 100\%); c) a fragilidade corpuscular média, correspondente à concentração de $\mathrm{NaCl}$ em que se observa $50 \%$ de hemólise.

\section{Cálculo das curvas de fragilidade osmótica eritrocitária}

A análise do probito (MEAD \& CURNOW, 1987) foi utilizada para calcular a FOE nas concentrações em que ocorreram as diferentes porcentagens de hemólise (H50 e H100), e foram representadas pelas curvas cumulativas. As curvas derivativas foram obtidas pela aplicação do princípio do "incremento hemolítico", no qual a quantidade adicional de hemólise que ocorre em cada tubo, seguindo o decréscimo da concentração de solução salina, foi colocada contra a respectiva concentração dessa solução.

\section{Análise Estatística}

Foram utilizados os testes não paramétricos de Kruskal-Wallis e de Dunn, sendo os cálculos processados no programa "Graphpad Instat".

\section{RESULTADOS}

Os resultados obtidos nos exames laboratoriais para avaliação do eritrograma e testes de função hepática e renal estão na tabela 1.

O resultado da análise estatística, em relação aos níveis séricos de triglicérides, mostrou diferença significante apenas entre os animais do grupo I (controle) e os animais ictéricos, que apresentaram níveis de triglicérides mais elevados ( $p<0,01)$, não havendo diferença entre os grupos de animais com insuficiência renal e aqueles com doença hepática. Quanto aos níveis de colesterol, observouse diferença significante entre os grupos I e II $(p<0,05)$, mas não houve diferença entre os animais ictéricos e aqueles com insuficiência renal. Em relação ao HDLcolesterol, detectou-se diferença estatística significante entre os animais do grupo controle comparativamente aos animais dos grupos II e III $(\mathrm{p}<0,05)$.

Os valores médios das concentrações de solução salina tamponada que correspondem a $50 \%$ e $100 \%$ de hemólise, verificados nos animais dos três grupos estão demonstrados na tabela 2 . O resultado da análise estatística não revelou diferença significante nas concentrações de solução salina em que se

Tabela 1 - Valores da média e desvio padrão dos parâmetros do eritrograma e bioquímica sérica dos animais do grupo I (controle), grupo II (insuficiência renal) e grupo III (doença hepática).

\begin{tabular}{|c|c|c|c|c|}
\hline Parâmetros & Grupo I & Grupo II & Grupo III & Valores normais \\
\hline Hemácias $\left(\mathrm{x} 10^{6} / \mu \mathrm{L}\right)$ & $9,39 \pm 1,62$ & $5,38 \pm 1,18$ & $6,93 \pm 1,54$ & $5,0-10,0$ \\
\hline Hematócrito (\%) & $43,2 \pm 7,02$ & $25,75 \pm 6,11$ & $32,22 \pm 10,20$ & $25-45$ \\
\hline Hemoglobina (g/dL) & $13,55 \pm 2,10$ & $8,5 \pm 2,5$ & $10,51 \pm 2,95$ & $8,0-17,0$ \\
\hline VCM (fl) & $46,22 \pm 3,89$ & $47,81 \pm 3,50$ & $46,09 \pm 8,46$ & $39,00-55,00$ \\
\hline CHCM (\%) & $31,45 \pm 1,71$ & $32,74 \pm 3,49$ & $33,11 \pm 4,06$ & $31-36$ \\
\hline Uréia (mg/dL) & $54,40 \pm 7,38$ & $435,00 \pm 108,89$ & $80,00 \pm 49,61$ & Até 40 \\
\hline Creatinina (mg/dL) & $1,44 \pm 0,40$ & $10,33 \pm 4,91$ & $1,55 \pm 0,47$ & $1,5-2,0$ \\
\hline Proteínas Totais $(\mathrm{g} / \mathrm{dl})$ & $7,91 \pm 0,79$ & $8,36 \pm 0,65$ & $7,69 \pm 1,18$ & $5,5-8,5$ \\
\hline Albumina $(\mathrm{g} / \mathrm{dl})$ & $3,03 \pm 0,52$ & $3,29 \pm 0,52$ & $2,78 \pm 0,89$ & $2,7-4,0$ \\
\hline $\operatorname{ALT}(\mathrm{U} / \mathrm{L})$ & $41,50 \pm 18,15$ & $35,00 \pm 25,79$ & $105,89 \pm 103,68$ & Até 50 \\
\hline AST (U/L) & $20,40 \pm 8,60$ & $33,13 \pm 32,76$ & $97,00 \pm 159,80$ & Até 40 \\
\hline FA (U/L) & $48,20 \pm 20,12$ & $27,13 \pm 14,37$ & $256,56 \pm 133,20$ & Até 70 \\
\hline GGT (U/L) & $1,10 \pm 0,31$ & $1,13 \pm 0,35$ & $3,11 \pm 3,02$ & Até 5 \\
\hline Bilirrubina Total(mg/dL) & - & - & $6,66 \pm 4,20$ & 0,15 a 0,20 \\
\hline B. Direta (mg/dL) & - & - & $3,89 \pm 2,60$ & Até 0,20 \\
\hline B. Indireta (mg/dL) & - & - & $2,77 \pm 2,49$ & Até 0,20 \\
\hline Triglicérides (mg/dL) & $63,44 \pm 22,24$ & $109,63 \pm 63,30$ & $146,67 \pm 76,55$ & $50-100$ \\
\hline Colesterol total (mg/dL) & $92,63 \pm 21,34$ & $236,38 \pm 123,64$ & $203,00 \pm 121,22$ & $75-250$ \\
\hline HDL-colesterol (mg/dL) & $63,2 \pm 14,21$ & $136,00 \pm 39,31$ & $100,86 \pm 51,32$ & $>35$ \\
\hline
\end{tabular}

VCM - volume corpuscular médio CHCM - concentração de hemoglobina corpuscular média

ALT - alanina aminotransferase AST - aspartato aminotransferase FA - fosfatase alcalina GGT - gama glutamiltransferase. 
Tabela 2 - Valores da média e desvio padrão das concentrações de solução salina tamponada $(\% \mathrm{NaCl})$ correspondentes a $50 \%$ e $100 \%$ de hemólise, do grupo I (controle), grupo II (insuficiência renal) e grupo III (doença hepática).

\begin{tabular}{llll}
\hline Parâmetros & Grupo I & Grupo II & Grupo III \\
\hline Hemólise 50\% & $0,59 \pm 0,06^{\mathrm{a}}$ & $0,60 \pm 0,06^{\mathrm{a}}$ & $0,61 \pm 0,07^{\mathrm{a}}$ \\
Hemólise 100\% & $0,30 \pm 0,04^{\mathrm{b}}$ & $0,47 \pm 0,06^{\mathrm{c}}$ & $0,39 \pm 0,09^{\mathrm{c}}$ \\
\hline
\end{tabular}

observou $50 \%$ de hemólise entre os três grupos $(\mathrm{p}>0,05)$, entretanto, a diferença foi relevante em relação às concentrações de solução salina em que se observou $100 \%$ de hemólise. Constatou-se que tanto os eritrócitos de animais ictéricos $(\mathrm{p}<0,05)$ quanto aqueles dos animais com insuficiência renal $(\mathrm{p}<0,001)$ apresentaram maior fragilidade osmótica frente a concentrações decrescentes de solução salina em relação ao grupo controle. Não houve diferença significante entre a fragilidade osmótica dos eritrócitos de animais com insuficiência renal e aqueles com doença hepática.

As curvas cumulativas e derivativas médias dos animais dos três grupos estão representadas nas figuras 1 e 2 , respectivamente.

\section{DISCUSSÃO E CONCLUSÃO}

Os resultados observados nos três grupos, em relação às concentrações de solução salina em que se observou $50 \%$ de hemólise, estão de acordo com os valores obtidos por outros autores, que obtiveram valor médio de 0,54\% para gatos sadios (JAIN, 1973; KOHN et al., 2000). Em relação a 100\% de hemólise, observou-se que, quando comparados ao grupo controle, os eritrócitos dos animais acometidos por insuficiência renal crônica apresentaram maior fragilidade osmótica, seguidos dos eritrócitos dos gatos portadores de afecção hepática. Não existem na literatura relatos de valores para a hemólise total frente a diferentes concentrações de solução salina na espécie felina, impossibilitando qualquer cotejamento.

Acredita-se que a fragilidade osmótica dos eritrócitos felinos, à semelhança do que ocorre em outras espécies, possa também ser influenciada por alterações metabólicas decorrentes de processos mórbidos.

SASAKI (1977) relatou aumento da fragilidade osmótica, decréscimo na quantidade de lipídios e decréscimo da taxa colesterol/fosfolípides

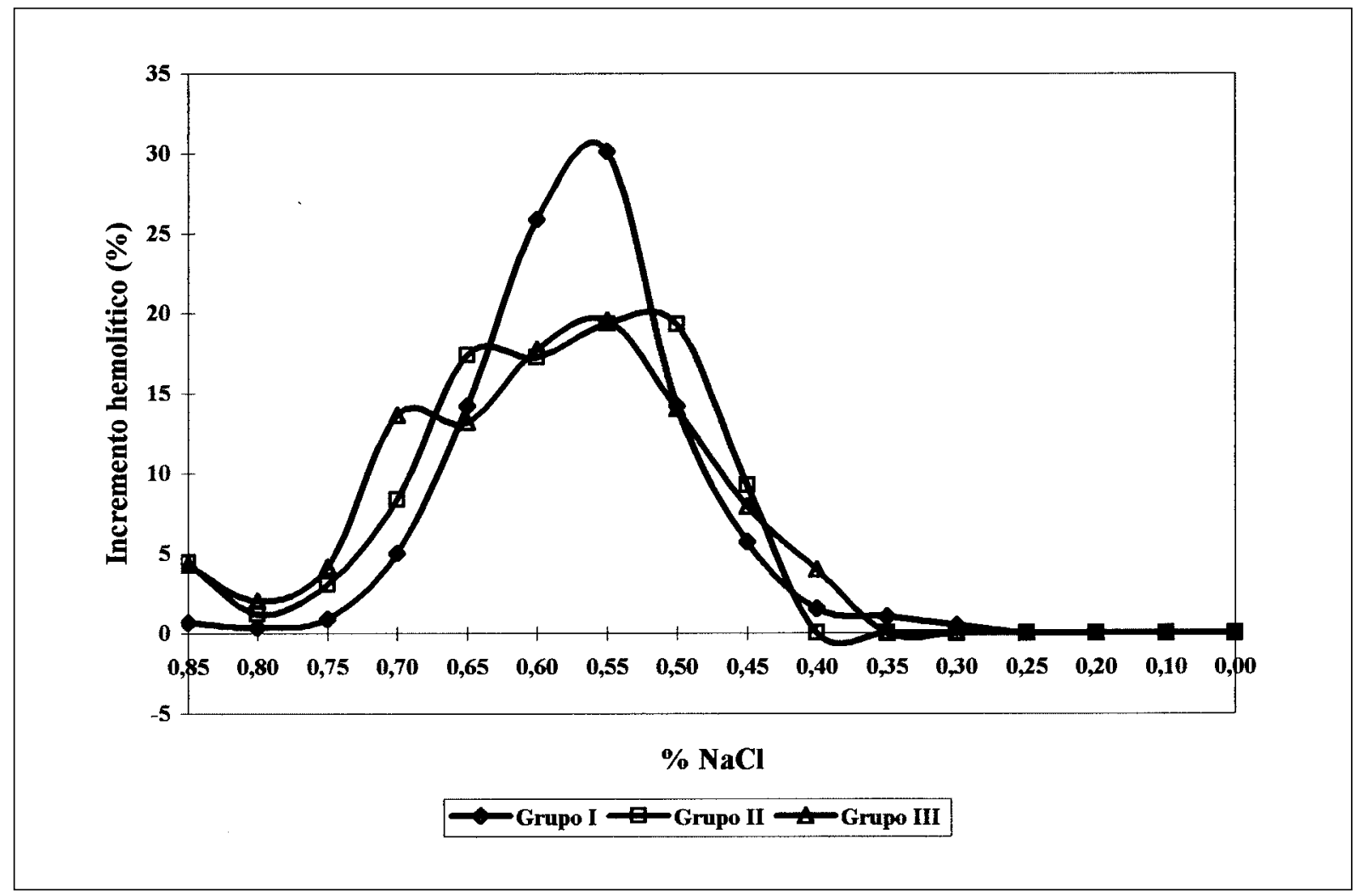

Figura 1 - Curvas cumulativas (médias) da fragilidade osmótica eritrocitária dos gatos do grupo I (controle), grupo II (insuficiência renal) e grupo III (doença hepática).

Ciência Rural, v.34, n.2, mar-abr, 2004. 


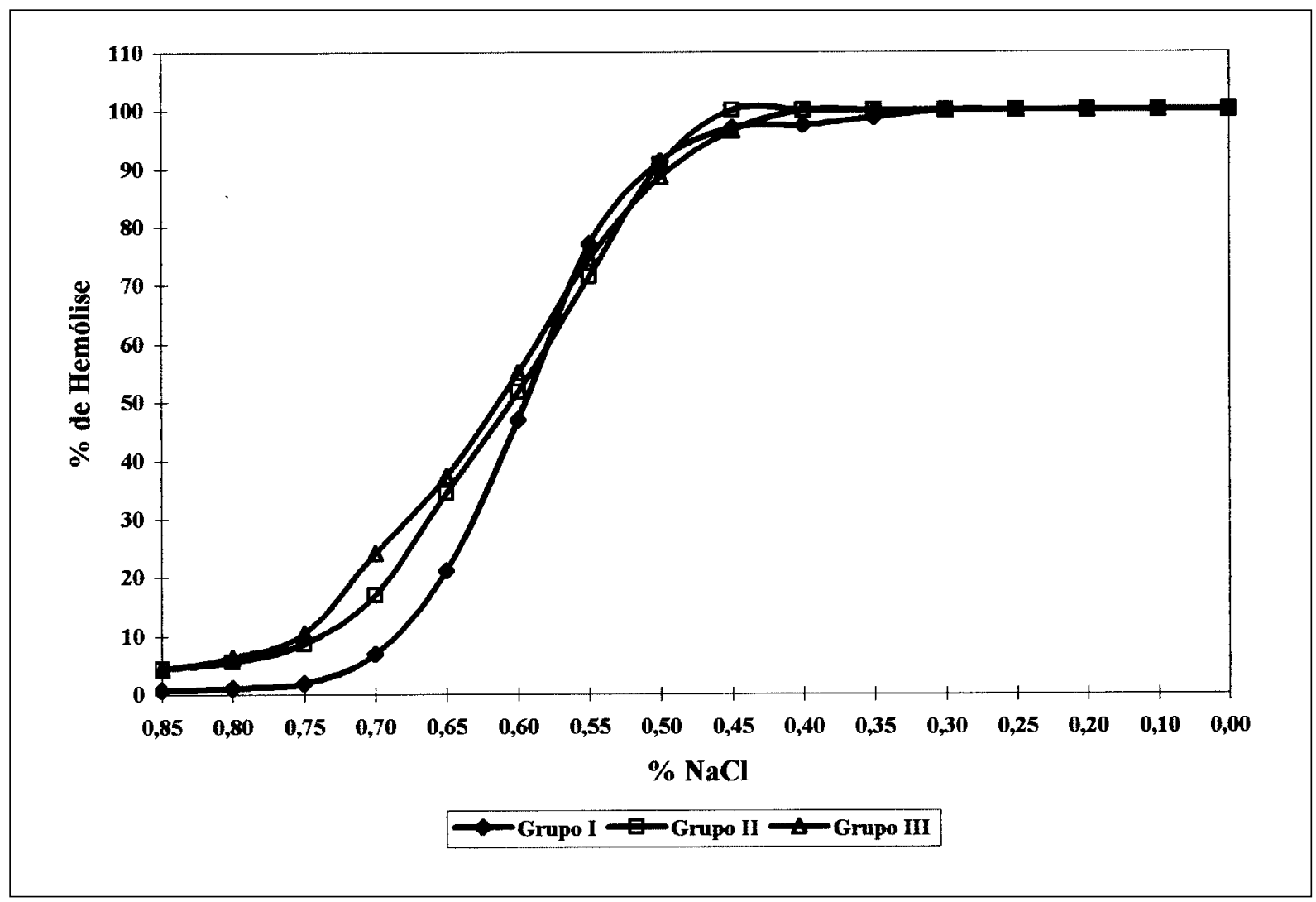

Figura 2 - Curvas derivativas (médias) da fragilidade osmótica eritrocitária dos gatos do grupo I (controle), grupo II (insuficiência renal) e grupo III (doença hepática).

da membrana eritrocitária em pacientes humanos urêmicos submetidos a hemodiálise, à medida que ocorria diminuição do colesterol livre na circulação. O autor observou uma correlação negativa entre a FOE e a taxa de colesterol livre nos eritrócitos. $\mathrm{O}$ oposto foi observado em casos de cirrose hepática, nos quais os eritrócitos apresentaram uma taxa de colesterol elevada, possivelmente decorrente da ausência da lecitina colesterol acil-transferase (LCTA) (OYABU et al., 1982).

Em pacientes humanos a fragilidade osmótica eritrocitária é influenciada pela quantidade de colesterol presente na membrana citoplasmática, tornando-se os eritrócitos mais resistentes à medida que aumenta o nível de colesterol sangüíneo (MAEDE, 1980). COOPER et al. (1975) relataram diminuição da FOE durante hepatopatias obstrutivas em pacientes humanos, devido ao aumento na relação superfície/ volume do eritrócito conseqüente à presença de colesterol intercalado na membrana eritrocitária e também à diminuição de atividade sérica de LCTA. SANTORO et al. (1994) verificaram um decréscimo na
FOE durante a leptospirose canina, sem a presença de anemia hemolítica, provavelmente relacionado aos distúrbios hepato-renais que ocorrem nessa afecção. No caso de cães, ZANUTTO et al. (1996) observaram alterações no metabolismo lipídico dos animais ictéricos, com aumento de colesterol, o que poderia estar relacionado ao decréscimo da FOE nesses animais.

Neste estudo, os níveis séricos de colesterol, triglicérides e HDL-colesterol foram significantemente mais elevados nos grupos de animais doentes, não havendo diferença significante entre os animais ictéricos e aqueles com insuficiência renal.

Quanto aos níveis séricos mais elevados de triglicérides e de colesterol nos animais ictéricos, estes podem ser indicativos da diminuição da esterificação dos triglicérides de origem exógena feita pelos hepatócitos e também pela diminuição da enzima LCTA, o que elevaria os níveis de colesterol. A hipercolesterolemia é um achado freqüente em gatos portadores de doença renal crônica, independentemente do tipo de doença renal primária 
(DIBARTOLA et al., 1987), ao contrário do que ocorre em outras espécies, inclusive no homem, em que o aumento de colesterol ocorre apenas em algumas condições específicas, como as glomerulopatias. Esses achados podem estar associados às particularidades do gato, de hábitos exclusivamente carnívoros e com seu metabolismo adaptado a um alto consumo de gordura.

Pelos resultados observados, não se pode inferir que, como em outras espécies, o aumento dos níveis de colesterol conduz à maior resistência dos eritrócitos, já que os animais com insuficiência renal e ictéricos apresentaram células mais frágeis do que os animais do grupo controle. Em relação aos animais com insuficiência renal, a acidemia observada freqüentemente nesses pacientes, pode justificar a menor resistência dos eritrócitos, não havendo nenhuma relação com os níveis séricos de colesterol. Entretanto, o mesmo não se pode afirmar em relação aos animais ictéricos. Nestes, a menor resistência dos eritrócitos pode estar relacionada a outras causas que não foram pesquisadas, bem como, em se tratando do gato, às diferenças no metabolismo lipídico, mais especificamente a hipercolesterolemia, atuando de forma única e peculiar sobre a membrana eritrocitária.

\section{REFERÊNCIAS BIBLIOGRÁFICAS}

CHRISTOPHER, M.M. Relation of endogenous Heinz bodies to disease and anemia in cats: 120 cases (1978-1987). Journal of American Veterinary Medical Association, v.194, n.8, p.1089-1095, 1989.

COOPER, R.A. et al. Modification of red cell membrane structure by cholesterol rich lipid dispersions. A model for the primary spur cell defect. Journal of Clinical Investigation, v.55, p.115-126, 1975.

DIBARTOLA, S. P. et al. Clinicopathologic findings associated with chronic renal disease in cats. Journal of American Veterinary Medical Association, v.190, p.1196-1202, 1987.

DIMOPOULlOS, G.T., BEDELL, D.M. Studies of bovine erythrocytes in anaplasmosis. American Journal of Veterinary Research, v.23, p.813-820, 1962.

JAIN, N.C. Osmotic fragility of erythrocytes of dogs and cats in health and in certain hematologic disorders. Cornell Veterinarian, v.63, p.411-423, 1973.
JAIN, N.C. Schalm's veterinary hematology. 4.ed. Philadelphia : Lea \& Febiger, 1986. 1221p.

KOHN, B. et al. Anemia, splenomegaly, and increased osmotic fragility of erythocytes in Abyssinian and Somali cats. Journal of the American Veterinary Medical Association, v.217, n.10, p.1483-1491, 2000.

MAEDE, Y. Studies on feline haemobartonellosis. VI. Changes of erythrocyte lipids concentration and their relation to osmotic fragility. Japanese Journal of Veterinary Science, v.42, n.3, p.281-288, 1980.

MAKINDE, M.O.; BOBADE, P.A. Osmotic fragility of erythrocytes in clinically normal dogs and dogs infected with parasites. Research in Veterinary Science, v.57, n.3, p.343-348, 1994.

MEAD, R.; CURNOW, R.N. Statistical methods in agriculture and experimental biology. 3.ed. New York : Chapman \& Hall, 1987. 335p.

OYABU, H.; TSUMOOTO, S.; KAGEYAMA, T. Clinical investigation on the osmotic fragility of erythrocytes in cirrhosis of the liver. Bulletin of the Osaka Medical School, v.28, p.33-45, 1982.

PERK, K.; FREI, Y.F.; HERZ, A. Osmotic fragility of red blood cells of young and mature domestic and laboratory animals. American Journal Veterinary Research, v.25, p.1241-1248, 1964.

PITTMAN, J.G.; MARTIN, D.B. Fatty acid biosynthesis in human erythrocytes: evidence in mature erythrocytes for an incomplete long chain fatty acid synthesizing system. Journal Clinical Investigation, v.45, p.165-172, 1966.

SANTORO, M.L.et al. Decreased erythrocyte osmotic fragility during canine leptospirosis. Revista do Instituto de Medicina Tropical de São Paulo, n.36, v.1, p.1-5, 1994.

SASAKI, S. Red cell lipids in patients with end-stage renal failure: relationship to osmotic fragility. Osaka City Medical Journal, v.23, n.1, p.85-98, 1977.

TELEN, M.J.; KAUFMAN, R.E. The mature erythrocyte. In: LEE, G.R. et al. Wintrobe's clinical hematology. Baltimore : Williams \& Wilkins, 1999. Cap.10, p.193-227.

WEED, R.I.; REED, C.F. Membrane alterations leading to red cell destruction. American Journal of Medicine, v.41, p.681-694, 1966.

ZANUTTO, M.S.et al. Fragilidade osmótica eritrocitária em cães com doenças hepática, renal e hepática e renal concomitante. In: CONGRESSO BRASILEIRO DE CLÍNICOS VETERINÁRIOS DE PEQUENOS ANIMAIS, 18., 1996, Olinda, PE. Anais... Olinda : Associação Nacional de Clínicos Veterinários de Pequenos Animais, v.1, p.163, 1996. 